\title{
DETERMINAÇÃO DAS CONDIÇÕES DE REAÇÃO DE POLIMERIZAÇÃO DE N-VINILPIRROLIDONA UTILIZANDO PLANEJAMENTO FATORIAL
}

\author{
D. B. MACHADO ${ }^{1}$, P. P FURTAT ${ }^{1}$ e R. A. F. MACHADO ${ }^{1}$ \\ ${ }^{1}$ Universidade Federal de Santa Catarina, Departamento de Engenharia Química e Engenharia de \\ Alimentos \\ E-mail para contato: diegobmach@gmail.com
}

\begin{abstract}
RESUMO - Dependo das especificações do produto, a poli(pirrolidona vinílica), possui diversas aplicações. Porém, a qualidade da mesma é facilmente comprometida se ocorrer pequenas variações durante a reação. Suas principais aplicações são na formulação de fármacos, cosméticos e na indústria de polímeros. Buscando uma otimização na reação de polimerização da n-vinilpirrolidona, adotou-se um planejamento fatorial $\left(3^{2}\right)$, trazendo como variáveis a interação entre dois iniciadores e temperatura de reação. Os iniciadores utilizados foram perpivolato de terc-butila e 2-etil-hexanoato de terc-butila. A resposta do planejamento é verificada através da massa molar e conversão. Através do perfil de valores preditos, utilizando software de estatística, tem-se como valor ótimo para reação a proporção 3:2 entre os iniciadores e temperatura de $60^{\circ} \mathrm{C}$. Como verificação, realizou-se a reação com os referidos valores, obtendo assim a confirmação dos valores de massa molar e conversão da polimerização.
\end{abstract}

\section{INTRODUÇÃO}

A poli(pirrolidona vinílica), ou PVP, é um polímero sintético hidroscópico e solúvel em água. O sucesso comercial da PVP é devido à sua compatibilidade biológica, não toxicidade, características adesivas e de formação de filmes, facilidade para formar complexos, comportamento relativamente inerte com relação a sais e ácidos, e sua resistência a degradação térmica em soluções (ROBISON et al., 1990). A PVP é um polímero vinílico, obtido através da polimerização vinílica via radicais livres, por meio aquoso ou em solventes orgânicos (NUYKEN; BILLIGPETERS, 1997).

Iniciadores como peróxido de hidrogênio ou peróxidos orgânicos geralmente são empregados em procedimentos de polimerização de n-vinilpirrolidona (NVP) fornecendo polímeros com diferentes massas molares, alguns procedimentos são apresentados por Cho e Barabas (1986) e Nuber, Denzinger e Sanner (1989).

Alguns dos desafios encontrados na polimerização da n-vinilpirrolidona é o controle da sua massa molar, que deve variar mediante o uso, e sua conversão. Segundo Rodrigues e Iemma (2005), o planejamento experimental condiz uma ferramenta poderosa para se chegar ás condições otimizadas de um processo, desenvolvimento de formulações de produtos ou simplesmente para avaliar efeitos ou 
impactos que os fatores têm nas respostas desejadas.

Desta forma, o presente trabalho, tem como objetivo utilizar da ferramenta de planejamento experimental para determinar as condições de reação da polimerização da n-vinilpirrolidona em solução. Foram determinadas duas variáveis de reação: a interação entre dois iniciadores e temperatura de reação, a fim de otimizar o processo de polimerização.

As respostas obtidas a partir do planejamento são dadas em termos de massa molar e conversão. Tendo em vista a possibilidade de fazer uso da viscosidade de soluções poliméricas para determinação de massa molar (BILLMEYER, 1984), os valores desta medida serão em valor de $K$, partindo dos procedimentos abordados por Büler (2001). Para a conversão utilizou-se do método gravimétrico.

\section{METODOLOGIA}

O monômero n-vinilpirrolidona foi fornecido pela empresa Termotecnica, com grau de pureza de 99\%, contendo como inibidor hidróxido de sódio (20ppm). Os iniciadores, adquiridos da Akzo Nobel, o utilizado na polimerização do NVP convencionalmente (CHO; BARABAS, 1986), perpivolato de terc-butila com pureza de $75 \%$ e oxigênio ativo de 9,18\% (AKZO NOBEL, 2005), e como iniciador teste, utilizou-se 2-etil-hexanoato de terc-butila com $97 \%$ de pureza e oxigênio ativo de 7,4\% (AKZO NOBEL, 2006).

Cho e Barabas (1986) assumem que para uma polimerização de NVP deve-se adotar a temperatura de $55^{\circ} \mathrm{C}$ e iniciador perpivolato de terc-butila ( $3 \mathrm{~mL}$ para $5 \mathrm{Kg}$ de massa reacional). As temperaturas adotadas foram 60,55 e $50^{\circ} \mathrm{C}$ e para contribuir à conversão pode-se adicionar um segundo iniciador (ARIOLI, 2004). As interações foram realizadas entre os iniciadores na proporção de 100\%-0, 80\%$20 \%$ e $60 \%-40 \%$, assim como segue na Tabela 1 .

Tabela 1 - Variáveis experimentais para reação de polimerização do NVP.

\begin{tabular}{llll}
\hline Variáveis & +1 & 0 & -1 \\
\hline A - Razão de iniciador $(\%)$ & 100 & 80 & 60 \\
$\mathrm{~B}-$ Temperatura $\left({ }^{\circ} \mathrm{C}\right)$ & 60 & 55 & 60 \\
\hline
\end{tabular}

As respostas do plenejamento, massa molar viscosimétrica e conversão, foram tratadas pelo Software Statistica da StatSoft.

\subsection{Reações de polimerização}

Nesta unidade experimental realizou-se a polimerização em solução. Utilizou-se para isso um reator do tipo batelada, encamisado, agitado, construído em vidro de borosilicato, com volume de 1000 $\mathrm{mL}$. A tampa do reator possui orifícios para as conexões necessárias do condensador, da haste do agitador e acesso ao meio reacional para controle da temperatura. Este controle de temperatura é realizado por meio do banho termostático, onde a água do mesmo circula em sistema fechado através de mangueiras conectadas à entrada e saída da camisa do reator. 
A agitação é realizada por um impelidor do tipo homogeneizador de aço inox com diâmetro total de $50 \mathrm{~mm}$, acoplado a um agitador magnético.

O procedimento para reação consistiu no carregamento de monômero, n-vinilpirrolidona, e água no reator, seguido de aquecimento, submetido à frequência de agitação de $150 \mathrm{rpm}$. Ao alcançar a temperatura estipulada pelo planejamento, estipula-se como tempo de 0 minuto de reação e adicionase o iniciador/iniciadores, segundo proporção estipulada também no planejamento. $\mathrm{O}$ tempo e reação foi mantido constante, em 180 minutos, e por fim, aquece-se a reação a um patamar da $80^{\circ} \mathrm{C}$ por 30 minutos, finalizando o processo de polimerização.

\subsection{Análise de massa molar viscosimétrica}

O equipamento utilizado na medida de viscosidades de soluções foi um viscosímetro capilar Ubbelohde. As medidas foram realizadas com o referido viscosímetro imerso em um banho com temperatura controlada em $25,0 \pm 0,1^{\circ} \mathrm{C}$. O tempo de escoamento, tanto do solvente quanto da solução, foi considerado como sendo a média dos tempos de três medidas consecutivas, com uma diferença máxima de 0,5 segundos. Todas as análises foram realizadas em triplicatas.

\subsection{Análise de conversão}

A conversão das reações foi determinada através de método gravimétrico, a partir dos valores de reagentes introduzidos na reação de polimerização. Para que a conversão seja máxima, a massa total de NVP deve ser polimerizada.

\section{RESULTADOS E DISCUSSÃO}

A apresentação dos resultados do planejamento fatorial encontra-se em duas partes, sendo os resultados de massa molar do polímero obtido (em valor de K-value) e a conversão da reação de polimerização. A Tabela 2 apresenta os resultados das noves reações de polimerização do NVP, sucessivo, a Tabela 3 e Tabela 4 expõem a análise de variância (ANOVA), com intervalo de confiança de $95 \%(\mathrm{p}<0,05)$, para as respostas do planejamento.

A partir dos dados de massa molar a Tabela 3, observa-se as interações das variáveis da reação, onde o valor de F mostra que o fator 'Temperatura' é mais significativo, concluindo que para a massa molar, portanto, a temperatura tem maior influência. De forma diferente, para a conversão (Tabela 4), o fator 'Razão de iniciadores' possui um controle mais significativo ao modelo. A interação das variáveis (Razão de iniciadores $x$ Temperatura) mostra-se com menor valor significativo em ambos modelos.

Os modelos estão dispostos nas equações 1 e 2, para massa molar e conversão, respectivamente. Onde RM é a variável 'Razão de iniciadores', em percentual de perpivolato de terc-butila e T é a variável 'Temperatura', em grau Celsius. 
Tabela 2 - Resultado do planejamento para o valor de massa molar viscosa e conversão.

\begin{tabular}{c|cc|cc}
\hline Experimento & A & B & Massa Molar & Conversão \\
\hline 1 & 0 & +1 & $105,9 \pm 1,1315$ & $0,960 \pm 0,002$ \\
2 & +1 & 0 & $109,7 \pm 1,178$ & $0,994 \pm 0,007$ \\
3 & -1 & -1 & $77,2 \pm 0,130$ & $0,899 \pm 0,009$ \\
4 & -1 & 0 & $77,8 \pm 0,824$ & $0,932 \pm 0,004$ \\
5 & +1 & +1 & $113,9 \pm 2,248$ & $1,021 \pm 0,001$ \\
6 & 0 & -1 & $75,7 \pm 0,380$ & $0,879 \pm 0,003$ \\
7 & +1 & -1 & $79,4 \pm 0,395$ & $0,942 \pm 0,001$ \\
8 & -1 & +1 & $89,9 \pm 0,395$ & $0,944 \pm 0,009$ \\
9 & 0 & 0 & $78,0 \pm 0,340$ & $0,882 \pm 0,003$ \\
\hline
\end{tabular}

Tabela 3 - Análise de variância (ANOVA) para massa molar viscosimétrica, modelo com correlação $\left(\mathrm{R}^{2}\right)$ de 0,87 .

\begin{tabular}{c|ccccc}
\hline Fatores & SS & df & MS & F & p \\
\hline Razão de iniciador L+Q & 1821,66 & 2 & 910,83 & 24,58 & 0,000003 \\
Temperatura L+Q & 3018,67 & 2 & 1509,34 & 40,73 & 0,000000 \\
$1 * 2$ & 352,52 & 1 & 352,52 & 9,51 & 0,005625 \\
Erro & 778,28 & 21 & 37,06 & & \\
Total SS & 5971,13 & 26 & & & \\
\hline
\end{tabular}

Tabela 4 - Análise de variância (ANOVA) para conversão da reação, modelo com correlação $\left(\mathrm{R}^{2}\right) \mathrm{de}$ 0,929 .

\begin{tabular}{c|ccccc}
\hline Fatores & SS & df & MS & F & p \\
\hline Razão de iniciador L+Q & 0,031 & 2 & 0,015 & 79,77 & 0,000000 \\
Temperatura L+Q & 0,021 & 2 & 0,011 & 55,48 & 0,000000 \\
$1 * 2$ & 0,001 & 1 & 0,001 & 4,266 & 0,051442 \\
Erro & 0,004 & 21 & 0,000 & & \\
Total SS & 0,057 & 26 & & & \\
\hline
\end{tabular}

$K-$ value $=445,99-4,41 \cdot R M+0,012 \cdot R M^{2}-9,98 \cdot T+0,075 \cdot T^{2}+0,054 \cdot R M \cdot T$

Conversão $=0,90+0,030 \cdot R M+0,048 \cdot R M^{2}+0,034 \cdot T+0,0046 \cdot T^{2}+0,0083 \cdot R M \cdot T$

A superfície de resposta do modelo aplicado à massa molar viscosimétrica, está apresentada na Figura 1a. Sabendo que a necessidade de utilização da PVP mais comum é quando sua massa molar está com valor entre 790.000 - 1.300.000, ou de 81 - 96 em valor de $K$-value, visualiza-se, essa região, situada no gráfico na faixa verde claro, onde $K$ é mais próximo do valor de 90 . Observa- 
se, ainda, o comportamento do gráfico com um crescimento mais explícito com o aumento da temperatura.

Subsequente, tem-se a superfície de resposta da conversão da reação de polimerização em solução, Figura 1b, mostra-se em temperaturas menores um valor de conversão menor e em maiores temperatura assumem valores de maior conversão. Ainda, para melhor conversão, a 'Razão de iniciador' em +1 , ou $60 \%-40 \%$, torna o valor de conversão, também, proveitoso.

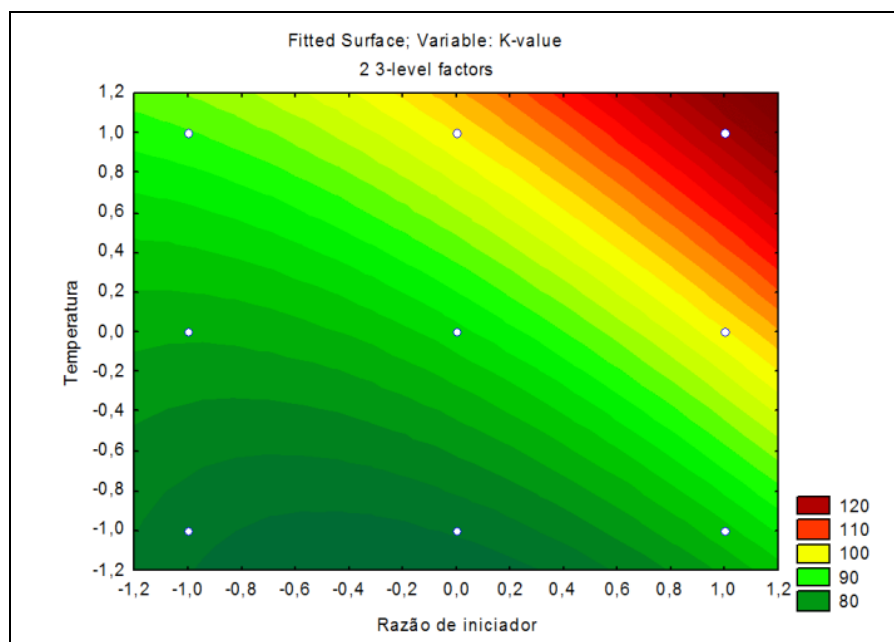

(a)

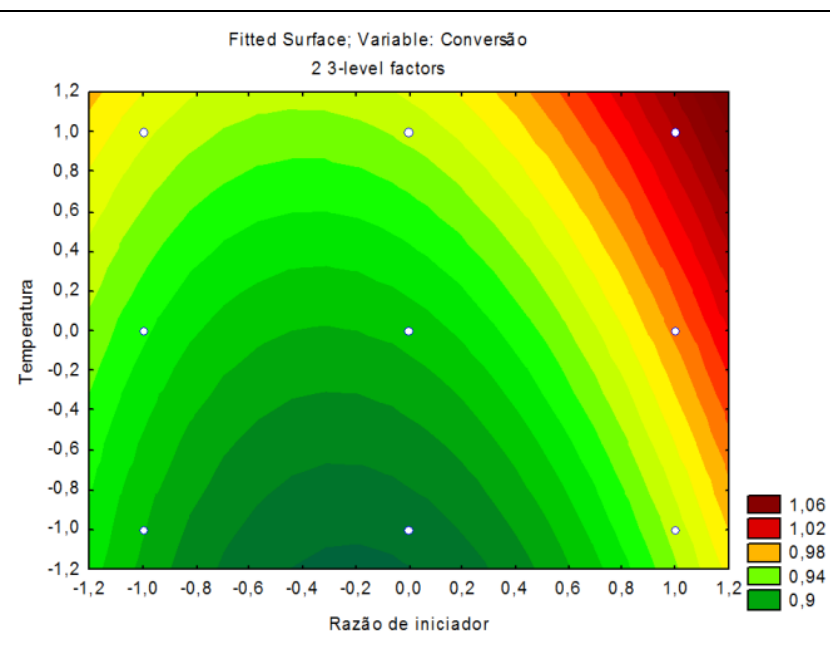

(b)

Figura 1 - Superfície de reposta do planejamento para a massa molar viscosimétrica do polímero formado (a) e conversão da reação (b).

Utilizando ainda de ferramenta estatística, fez-se a análise de influência mútua dos fatores (Razão de iniciador e Temperatura), com um gráfico de valores preditos. Este estudo aplica uma interpolação do tipo Spline e otimiza um ponto dentro do planejamento. Para tanto, a Figura 2, apresenta os perfis de valores preditos, para a massa molar viscosimétrica próxima de 90 e conversão próxima a máxima. Assim, os pontos $(+1,0 ;-0,5)$ e $(-1,0 ;+1,0)$ convergem seus valores a uma otimização do planejamento.

Dentre os experimentos do planejamento, um dos pontos de otimização tornar-se o já aplicado, ponto $(-1,0 ;+1,0)$, que apresenta valor de 89,9 e 0,944 , para massa molar e conversão, respectivamente. Portanto, realizou-se o experimento para o ponto de otimização $(+1,0 ;-0,5)$, onde a reação de polimerização ocorre com $100 \%$ de massa de perpivolato de terc-butila e temperatura de $52,5^{\circ} \mathrm{C}$. Os dados de valores preditos e dos experimentos encontram-se na Tabela 5.

Observando os dados de análise para os pontos de otimização, pode-se concluir que a interpolação do tipo Spline torna-se adequada, contendo os valores de respostas equivalentes aos dados preditos, desta forma, o erro entre as respostar apresenta valores baixos. 


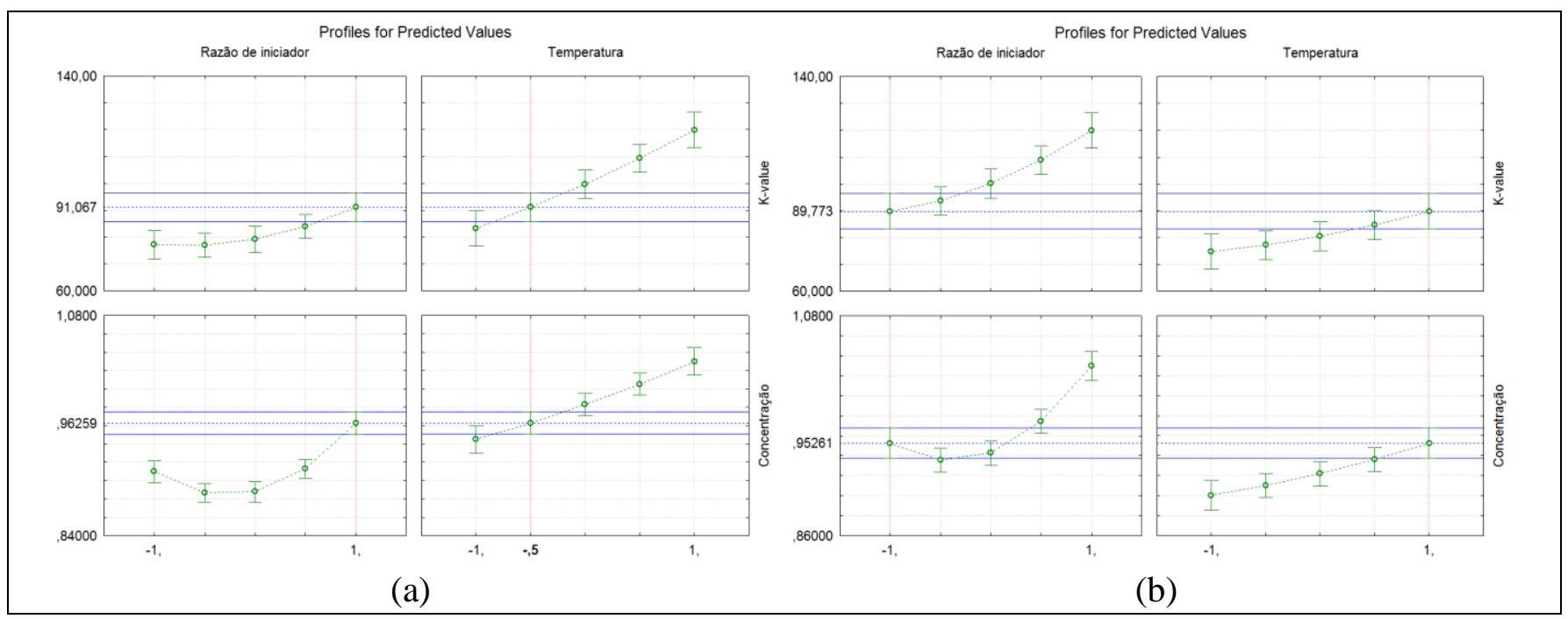

Figura 2 - Gráfico de interação entre respostas para ponto $(+1,0 ;-0,5)($ a) e ponto $(-1,0 ;+1,0)$ (b).

Tabela 5 - Resultados comparativos para pontos de otimização.

\begin{tabular}{clcc}
\hline \multicolumn{2}{c}{ Ponto de otimização } & Massa molar $($ K-value $)$ & $\begin{array}{c}\text { Conversão de } \\
\text { reação }\end{array}$ \\
\hline \multirow{2}{*}{$(+1,0 ;-0,5)$} & $\begin{array}{l}\text { Valor predito } \\
\text { Reação de } \\
\text { polimerização }\end{array}$ & 91,1 & 0,963 \\
& Erro (\%) & $90,8 \pm 0,442$ & $0,968 \pm 0,003$ \\
\hline \multirow{2}{*}{$(-1,0 ;+1,0)$} & $\begin{array}{l}\text { Valor predito } \\
\text { Reação de } \\
\text { polimerização }\end{array}$ & 0,330 & $-0,516$ \\
\cline { 2 - 4 } & Erro (\%) & 89,8 & 0,953 \\
& & $-0,111$ & $0,944 \pm 0,009$ \\
\hline
\end{tabular}

\section{CONCLUSÃO}

No presente trabalho utilizou-se um planejamento fatorial, onde a utilização desta ferramenta estatística mostra-se atraente para os estudos experimentais, havendo a capacidade de analisar os efeitos dos fatores que influenciam nas respostas e otimizar os ensaios laboratoriais.

Desta forma, foi possível concluir que a massa molar do polímero possui maior efeito a partir das variações de temperatura, seguido da interação entre os iniciadores, que mesmo com efeito menor, também influencia nas respostas. Dos efeitos observados na conversão, a interação entre iniciadores mostra-se mais influente.

Ainda, com o apoio estatístico, pode-se concluir que os pontos de otimização tiveram os valores preditos e de reações de polimerização com respostas equivalentes, podendo estimar as respostas de massa molar e de conversão. 


\section{REFERÊNCIAS}

AKZO NOBEL. Product Data Sheet: Trigonox ${ }^{\circledR}$ 21S. Amersfoort, The Netherlands, 2006.

AKZO NOBEL. Product Data Sheet: Trigonox ${ }^{\circledR}$ 25-C75. Amersfoort, The Netherlands, 2005.

ARIOLI, R. Utilização de iniciadores multifuncionais na polimerização do estireno em suspensão. Dissertação (Mestrado) - Universidade Federal de Santa Catarina, Centro Tecnológico, Programa de Pós-Graduação em Engenharia Química, Florianópolis, SC, 2004.

BILLMEYER, F. W. Textbook of polymer science. United States of America: John Wiley \& Sons, Inc., 1984.

BÜLER, V. Kollidon: Polyvinylpyrrolidone for pharmaceutical industry. 6.ed. Germany: [s.n.], 2001.

J R Cho e R S Barabas. Preparation of polyvinylpyrrolidone or vinylpyrrolidone/vinyl acetate copolymers of various molecular weights using a single initiator system. 1986. n. patente US 1211892.

NUYKEN, O.; BILLIG-PETERS, W. Polystyrenes and other poly(vinyl compound)s. [S.1.]: Handbook of Polymer Synthesis:Secund Edition, 1997.

ROBISON, B. V. et al. PVP: a critical review of the kinetics and toxicology of polyvinylpirrolidone. London: Lewis Publishers, 1990.

RODRIGUES, M. I.; IEMMA, A. F. Planejamento de experimentos e otimização de processos: Uma estratégia sequencial de planejamentos. [S.1.]: Casa do Pão Editora, 2005. 